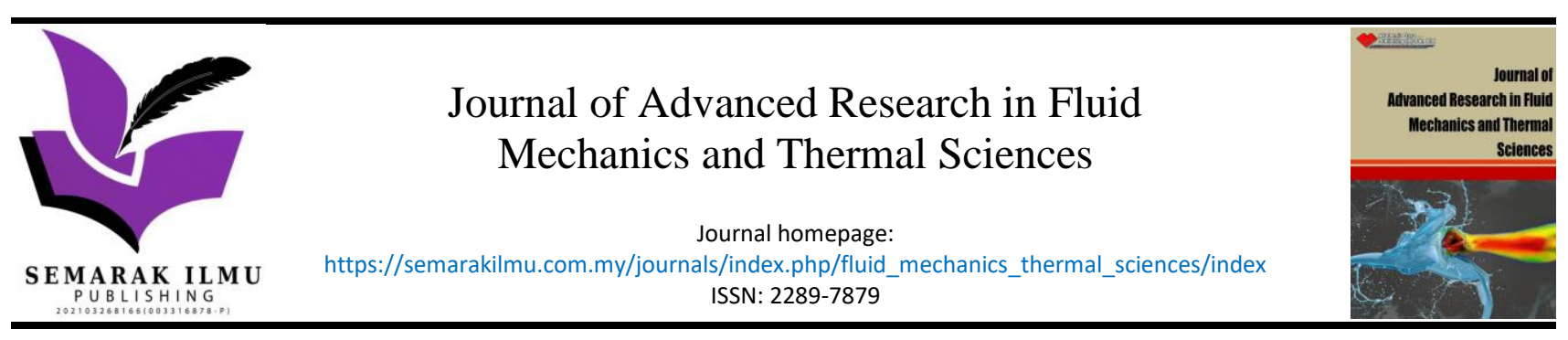

\title{
Application of Gaussian Process Regression (GPR) in Gas Hydrate Mitigation
}

\author{
Sachin Dev Suresh ${ }^{1}$, Ali Qasim ${ }^{1,2}$, Bhajan Lal ${ }^{1,2, *}$, Syed Muhammad Imran ${ }^{3}$, Khor Siak Foo ${ }^{1,4}$ \\ Chemical Engineering Department, Universiti Teknologi PETRONAS, Bandar Seri Iskandar, 32610, Perak, Malaysia \\ $\mathrm{CO}_{2}$ Research Centre (CO 2 RES), Universiti Teknologi PETRONAS, Bandar Seri Iskandar, 32610, Perak, Malaysia \\ Department of Chemical Engineering, COMSATS University Islamabad, Lahore, Pakistan \\ PTTEP, Level 26-30, Tower 2, Petronas Twin Towers, Kuala Lumpur City Centre, 50088, Kuala Lumpur, Malaysia
}

\section{Article history:}

Received 8 July 2021

Received in revised form 19 September 2021

Accepted 23 September 2021

Available online 26 October 2021

\begin{abstract}
The production of oil and natural gas contributes to a significant amount of revenue generation in Malaysia thereby strengthening the country's economy. The flow assurance industry is faced with impediments during smooth operation of the transmission pipeline in which gas hydrate formation is the most important. It affects the normal operation of the pipeline by plugging it. Under high pressure and low temperature conditions, gas hydrate is a crystalline structure consisting of a network of hydrogen bonds between host molecules of water and guest molecules of the incoming gases. Industry uses different types of chemical inhibitors in pipeline to suppress hydrate formation. To overcome this problem, machine learning algorithm has been introduced as part of risk management strategies. The objective of this paper is to utilize Machine Learning (ML) model which is Gaussian Process Regression (GPR). GPR is a new approach being applied to mitigate the growth of gas hydrate. The input parameters used are concentration and pressure of Carbon Dioxide $\left(\mathrm{CO}_{2}\right)$ and Methane $\left(\mathrm{CH}_{4}\right)$ gas hydrates whereas the output parameter is the Average Depression Temperature (ADT). The values for the parameter are taken from available data sets that enable GPR to predict the results accurately in terms of Coefficient of Determination, $\mathrm{R}^{2}$ and Mean Squared Error, MSE. The outcome from the research showed that GPR model provided with highest $\mathrm{R}^{2}$ value for training and testing data of $97.25 \%$ and $96.71 \%$, respectively. MSE value for GPR was also found to be lowest for training and testing data of 0.019 and 0.023 , respectively.
\end{abstract}

\section{Keywords:}

Gas Hydrate; Gaussian Process Regression; Least Square Support Vector Machine; Artificial Neural Network; Average Depression Temperature

\section{Introduction}

Gas hydrate was developed in a pipeline where many efforts have been made in the 1930 s to understand the clathrate hydrate in terms of structure, compound, balance state and way of managing them due to it poses issues such as plugging of pipelines to produce offshore oil and gas, pressure drop and corrosion plants [1-7]. When sufficient water molecules in the form of oil and hydrocarbon vapour crystallize under intermittent flow conditions, gas hydrate or known as clathrate

\footnotetext{
* Corresponding author.

E-mail address: bhajan.lal@utp.edu.my
}

https://doi.org/10.37934/arfmts.88.2.2737 
hydrate occurs. It is an ice-like, non-stochiometric crystalline mixture made up of a water cage frame dominated by gas molecules like methane, $\mathrm{CH}_{4}$, ethane, $\mathrm{C}_{2} \mathrm{H}_{5}$, and carbon dioxide, $\mathrm{CO}_{2}$, that forms solid particles that takes place under high pressure and low temperature conditions [3-6]. Gas hydrates are produced in the petroleum and natural gas industries' output, refining and transmission facilities [8-10].

The gas hydrates are formed in 3 structures, which are I, II and $\mathrm{H}$, based on the form and size of guest molecules [7]. Structure I, structure II, and structure $\mathrm{H}$ at which smallest and largest formers comprised of mixtures of methane, propane, nitrogen known as type $\mathrm{II}, \mathrm{CO}_{2}$ and $\mathrm{CH} 4$ form type I and methyl cyclopentane formers as type $\mathrm{H}$ formers are typically formed gas hydrate structures [11-13]. Structure I unit cells contain eight cavities (two smalls and six wide ones) and are formed by 46 water molecules bound to hydrogen. Besides that, there are 136 water molecules for a unit cell of structure II and enclosed 24 cavities, including 16 smalls and 8 wide ones [14]. It is therefore important to resolve the problem of gas hydrate development by introducing appropriate preventive and predictive strategies $[3,16]$. There are many risk control techniques that have been used, such as the use of thermodynamic hydrate inhibitor (THI) inhibitors and low-dose hydrate inhibitor (LDHI) inhibitors. Both chemical inhibitors play a key role in preventing gas hydrate development in the pipelines of the oil and gas industry. Two commercially used thermodynamic hydrate inhibition compounds are methanol (MeOH) and monoethylene glycol (MEG), while kinetic hydrate inhibitors $(\mathrm{KHI})$ and anti-agglomerants (AAs) are two types of low-dose hydrate inhibitors $[17,18]$. The purpose of THIs, also like water-ice antifreezes, is to disrupt the mechanism in which hydrates develop. In conjunction, the function of $\mathrm{KHI}$ is to delay crystallisation of hydrate and the function of AAs is to promote distortion of hydrate molecules [10]. Conversely, because of improvement in deep-sea pipeline placement, the use of THIs is not effective because it creates high operational and capital expense (CAPEX) relative to LDHIs that are more cost-effective $[1,13]$.

Advance management approach is the implementation of expertise focused on predictive study of multiphase flow, fluid chemistry, hydrate kinetics and plugging processes that do not require power calculation [10]. The use of Machine Learning (ML) is an innovative risk control technique that is currently being applied. ML is basically a numerical representation of a phenomenon, given with a certain significance and based on a certain environment, aimed at performing a job [8]. Deployment of ML models would allow us to do predictive analysis of gas hydrate growth because the value of determining hydrate formation is also that the solid crystalline structure which forms like ice will plug the oil and gas pipes as hydrate forms, either during gas production or transmission. This presents a potentially expensive situation for the organisation as it often takes additional time and cost to conduct a corrective intervention. Laboratory experiments are required to determine at which conditions for hydrate to perform. The best practise, therefore, is to predict the formation of hydrates by using the ML model to minimise gas hydrate growth.

\section{Methods and Theory}

A universal workspace is called Machine Learning, ML, ready to be used for classification problems, identifying a fitting category for a new set of outcomes, and regression tasks that estimate the relationship between data groups given. $\mathrm{ML}$ is essentially a numerical description of a phenomenon aimed at completing a job, assigned a certain value and based on a certain environment. The ML system can be referred to as knowledge discovery in the database (KDD) which focuses on the creation of algorithms and the test cycle. Initially, ML models are built in a training step where data is collected from historical datasets and pre-processed to delete or normalise outliers, manage incomplete data, search, and aggregate parameters. The next step is data migration, 
collection of suitable data attributes, minimization of redundancies, adaptation of data format to the specified task, which is a critical step in the ML workflow [14]. The algorithms can be further divided into three distinct families of learning that are supervised learning, unsupervised learning, and reinforcement learning. Molecular modelling and statistical algorithms such as quantitative structure-activity relationship (QSAR) models have previously been used in drug industry [15].

Throughout this study, supervised learning will be based on the most widely used machine learning algorithm, wherein the input data is referred to as training data and has a predetermined mark for predicting predetermined output. Based on whether discrete or continuous output features are used, supervised learning can be further divided into classification and regression activities [14]. Several existing ML models, such as Artificial Neural Network (ANN), Gaussian Process Regression (GPR) and other existing ML models, are present in the mitigation of gas hydrate formation [16-27].

\subsection{Least Square Version of Support Vector Machine (LSSVM)}

LSSVM development aims to reduce the complexity of the Support Vector Machine (SVM) and eradicate ambiguity in the optimization phase by deciphering linear formulas using linear coding rather than solving quadratic encoding equations. LSSVM algorithm's output can be overcome by fixed linear equality rather than inequality constraints $[13,28]$. General formulation that being used in approximating a given data set $\left\{\left(x_{1}, y_{1}\right),\left(x_{2}, y_{2}\right), \ldots,\left(x_{N}, y_{N}\right)\right\}$ into consideration by employing following nonlinear function [29]:

$f(x)=\langle w, \Phi(\mathrm{x})\rangle+b$

where $\langle.,$.$\rangle represent dot product; \Phi(\mathrm{x})$ denotes the nonlinear function that performs regression; $b$ and $\mathrm{w}$ are bias terms and weight vector, respectively. The LSSVM model structure can be seen in Figure 1 where the $\mathrm{K}\left(\mathrm{x}_{\mathrm{i}}, \mathrm{x}_{\mathrm{j}}\right)$ is defined as kernel function [23].

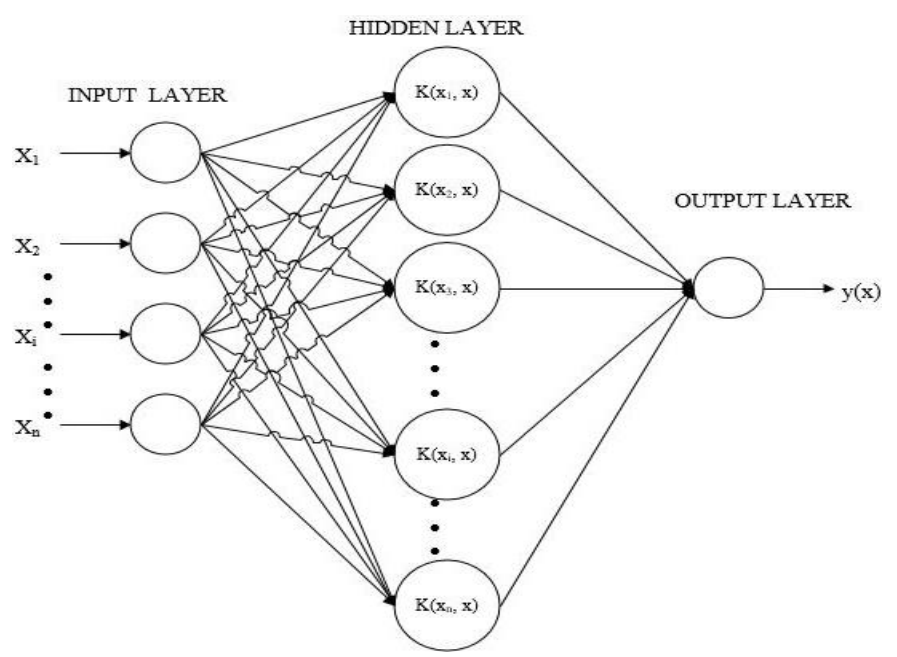

Fig. 1. LSSVM model architecture

\subsection{Artificial Neural Network (ANN)}

ANN is a measurement tool based on how measurements are carried out by the brain $[19,23]$. It has been developed for a wide range of topics, such as classification, approximation of functions and prediction [25-27]. Inside and between the layers, there are several similarities showing the strength and weights between neurons that can be seen in Figure 2 . 
Output of the ANN assuming a linear output neuron $j$, a single hidden layer with $\mathrm{h}$ sigmoid hidden nodes and the output variable $\left(x_{t}\right)$ is given by [23]:

$x_{t}=g\left(\sum_{j=1}^{h} w_{j} f\left(s_{j}\right)+b_{k}\right)$

where $g($.$) is the linear transfer function of the output neuron k$ and $b_{k}$ is its bias, $w_{j}$ is the connection weights between hidden layers and output units, $f($.$) is the transfer function of the hidden layer.$

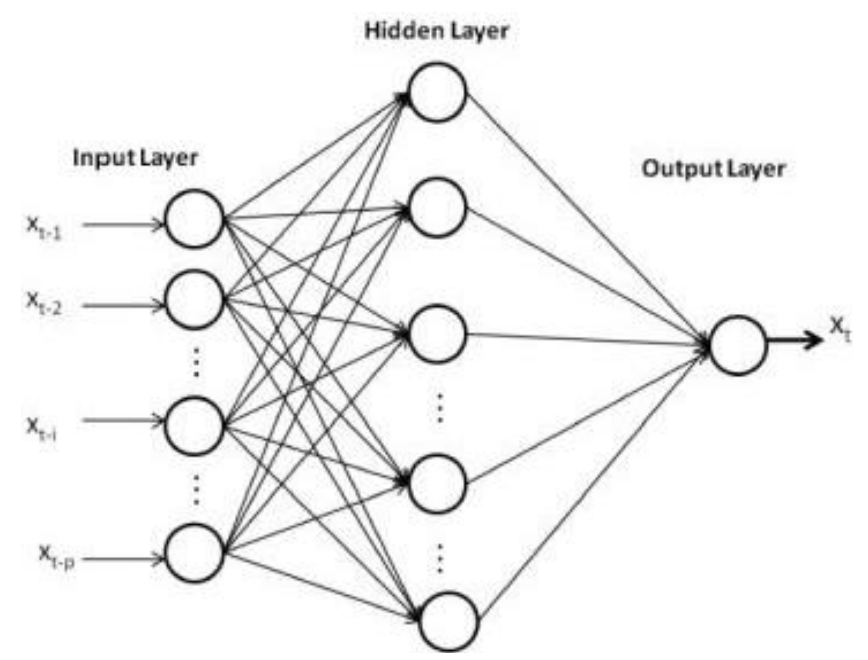

Fig. 2. Framework of ANN

\subsection{Gaussian Process Regression (GPR)}

GPR can be a valuable method to dynamically perform inference where it defines the maximum possible output based on given data sets and performs precise function approximation in highdimensional space [30-35]. GPR is a non-parametric Bayesian approach to the issue of regression by using Bayesian inference to capture a wide spectrum of relations between input and output [36-48]. The general formulation for GPR, based on Bayesian analysis, can be seen from Eq. (3) [32]:

$y=f(x)+\epsilon$

Where $f$ is the function value, $y$ is the observed target value, $\epsilon$ is the additive noise which is independent and adhere to normal distribution with zero mean, and variance, $\sigma_{n}^{2}$ as seen from the Eq. (4).

$\epsilon \sim \mathcal{N}\left(0, \sigma_{n}^{2}\right)$

This will be written in matrix algebra as [31]:

$y=x^{T} w+\epsilon$

where $x^{T}$ is the input vector and $w$ is the vector of weights (parameters) of the linear model. The formula to find $w$ is stated in Eq. (6):

$w \sim \mathcal{N}\left(0, \sum p\right)$ 
which it adheres to Gaussian prior with covariance matrix, $\sum p$. The Bayesian linear model's inferences are based on the posterior distribution over the weights, determined by the Bayes law, as can be seen in Eq. (7) and Eq. (8) [31].

posterior $=\frac{\text { likelihood } x \text { prior }}{\text { marginal likelihood }}$

$p(w \mid y, X)=\frac{p(y \mid X, w) p(w)}{p(y \mid X)}$

In addition to that, the newest solution is found to be the GPR application in mitigating the growth of the gas hydrate. The concentration and pressure of the $\mathrm{CH}_{4}$ and $\mathrm{CO}_{2}$ in the hydrated gas are the inputs to be used. Whereas average depression temperature (ADT) will be the output of the GPR. The results obtained will be evaluated in terms of Determination Coefficient, $R^{2}$, and Mean Squared Error, MSE. Then the $\mathrm{R}^{2}$ and MSE values will be compared with other ML models. The following equation can be used as the formulation for $\mathrm{R}^{2}$ and MSE:

$R^{2} \%=100\left(1-\frac{\sum_{i}^{n}\left(o_{i}-t_{i}\right)^{2}}{\sum_{i}^{n}\left(o_{i}-\text { average }\left(t_{i}\right)\right)^{2}}\right)$

$M S E \%=100\left(\frac{\sum_{i}^{n}\left(o_{i}-t_{i}\right)^{2}}{N}\right)$

where $\mathrm{n}$ denotes the number of samples; $o_{i}$ and $t_{i}$ are the predictions of the model and corresponding targets, respectively.

The output which is the ADT is the difference between the temperature of the pure water and temperature of the inhibitors mixed with the pure water. Below demonstrates the equation used to calculate ADT [40]:

$A D T=\frac{1}{n} \sum_{i=1}^{n} \Delta T$

where $\mathrm{n}$ is the number of data point, $\Delta T$ is the difference between the equilibrium point of temperature in presence of pure water and hydrate inhibitors at a specified pressure, MPa and concentration, wt\%.

\section{Results and Discussion}

Applied different Machine Learning (ML) models such as Least Square Support Vector Machine (LSSVM), Artificial Neural Network (ANN) and Gaussian Process Regression (GPR) to predict the output which is the Average Depression Temperature and inputs are Pressure and Concentration. The data generated from the 140 data points were utilized to plot the Predicted results of ADT versus Experimental results of ADT for each respective ML models. From the 140 data points, 112 data points will be used as training set and remaining 28 data points will be utilized as testing set. The result of training, testing prediction for each $\mathrm{ML}$ models in the work are presented.

From the Figure 3 , it can be observed that results from the training and testing data to obtain the predicted analysis values of ADT with the experimental values of ADT in terms of ${ }^{\circ} \mathrm{C}$. This GPR model is constructed with kernel present in it. Kernel is a function that represents feature space and hence, naturally incorporate the similarity measure between data points. Therefore, most used kernel that 
has been implemented in this GPR model is Radial Basis Function (RBF) kernel [31]. The length-scale, $\lambda$ of the RBF kernel used is 10 . This value is embedded to ensure the smoothness of the prediction of the output which is the ADT.

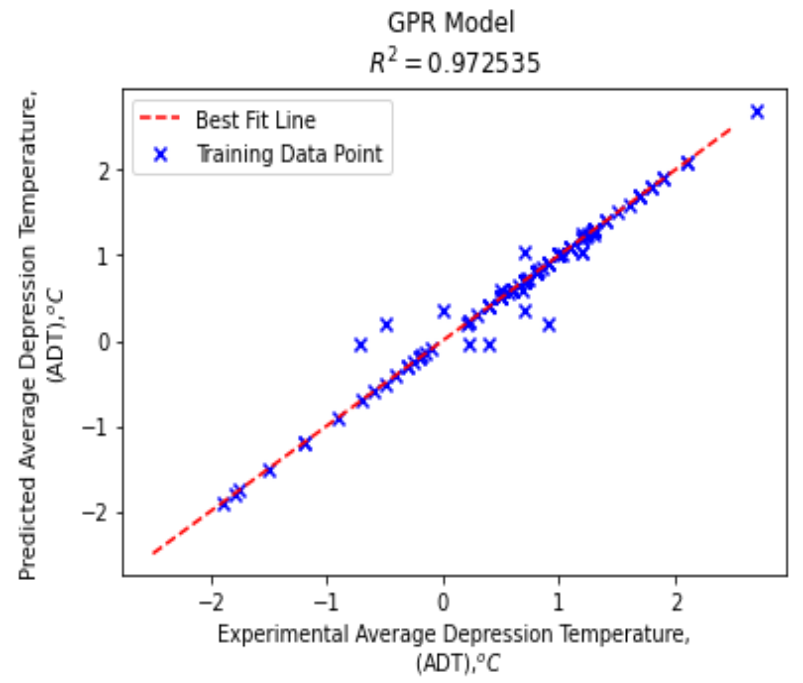

(a)

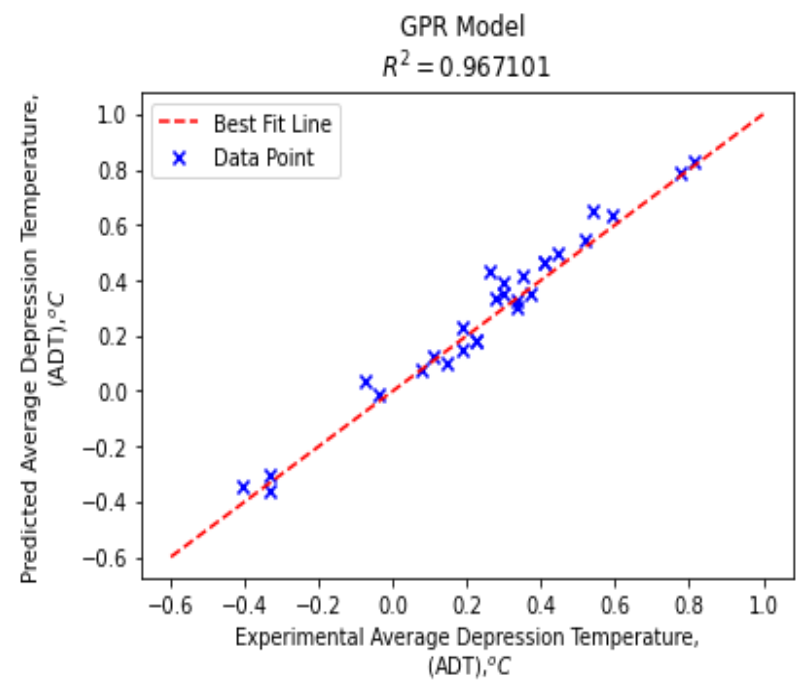

(b)

Fig. 3. GPR model outcomes for (a) training and (b) testing sets

From Figure 4, the results obtained by the predicted ADT versus the experimental ADT gives coefficient of determination for testing and training set approximately $96 \%$ and $95 \%$ respectively. In the ANN model, the hyperparameter which has been applied is Lbfgs optimizer because due to a smaller number of datasets and gives fast iterations. Lbfgs optimizer employs Quasi-Newton method where the results obtained from this optimizer will converge faster and perform better. The hidden layer size that has been implemented is 6 . Nonetheless, this ANN model does gives various local minimum value rather than global minimum value thus more fluctuations of the results tend to occur leading to $\mathrm{R}^{2}$ and MSE values not to be stable and accurate.

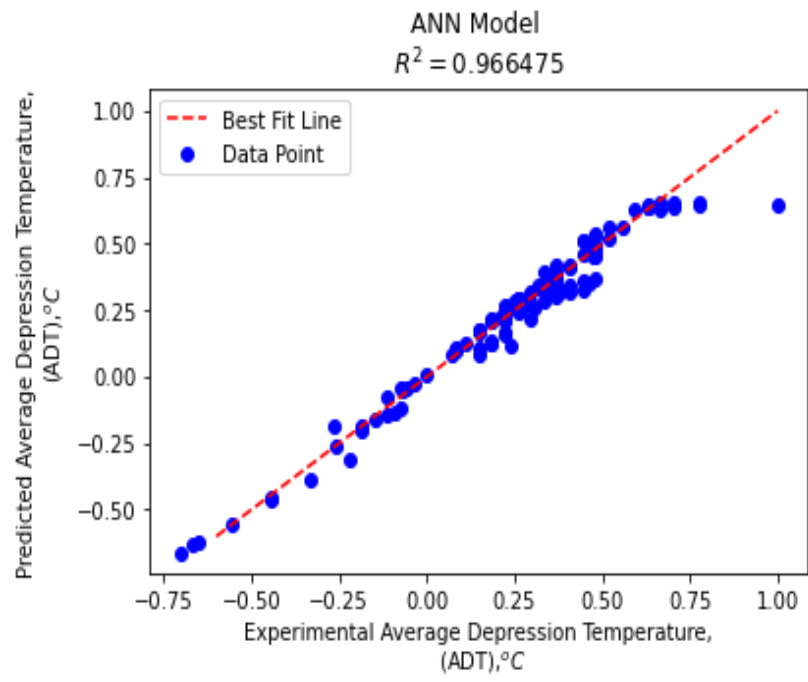

(a)

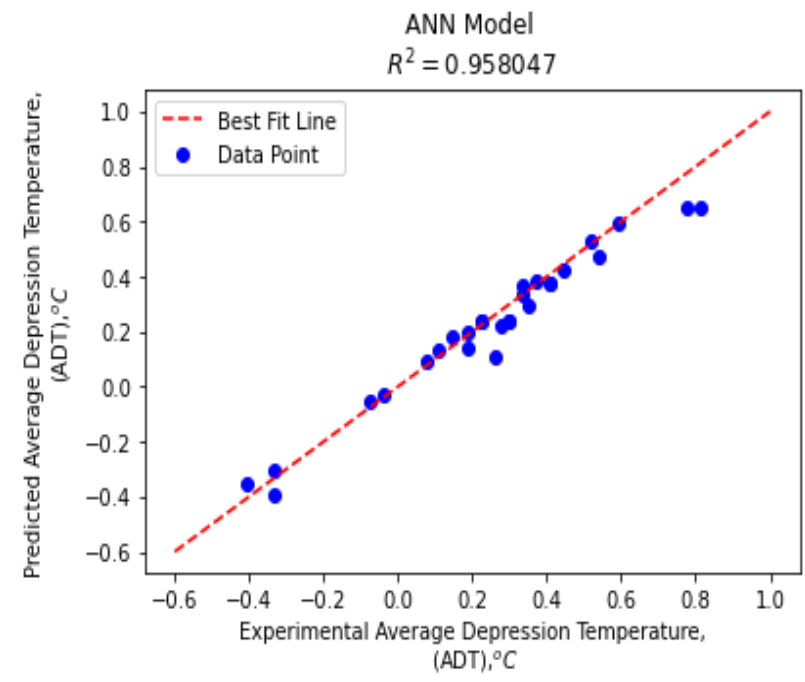

(b)

Fig. 4. Performance of ANN for (a) training and (b) testing sets 
From Figure 5, shows the results obtained by the LSSVM model for the training and test set with the value of $-0.06 \%$ and $0.131 \%$, respectively. Hence, this indicates that the predicted value of ADT does not correspond well to the experimental values of ADT in which variance can be observed as well as number of iterations applied is 6000.This is due to LSSVM model is an embedded with linear programming to solve the regression analysis. With this small dataset, this LSSVM model is prone to overfitting consequently leading to obtaining inaccurate coefficient of determination and higher value of mean squared error.

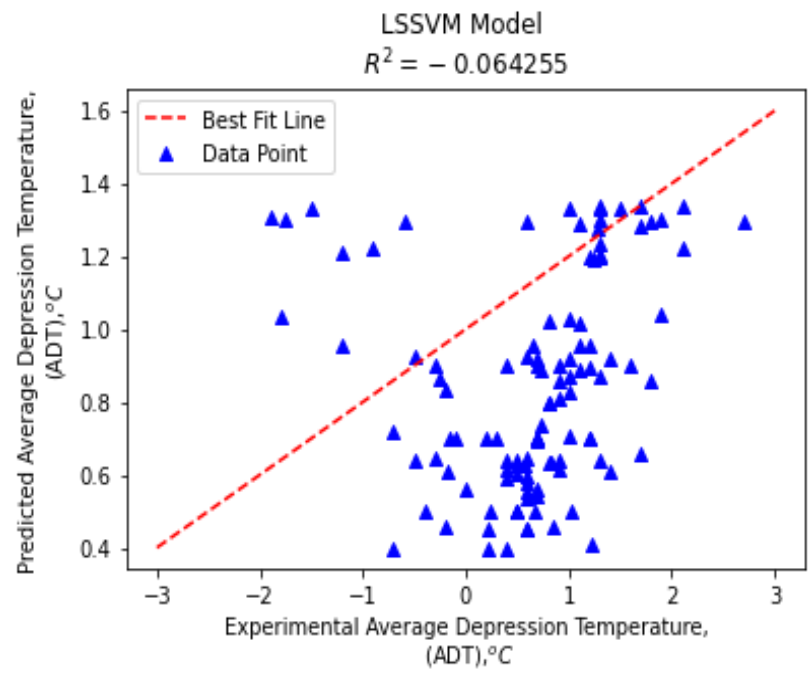

(a)

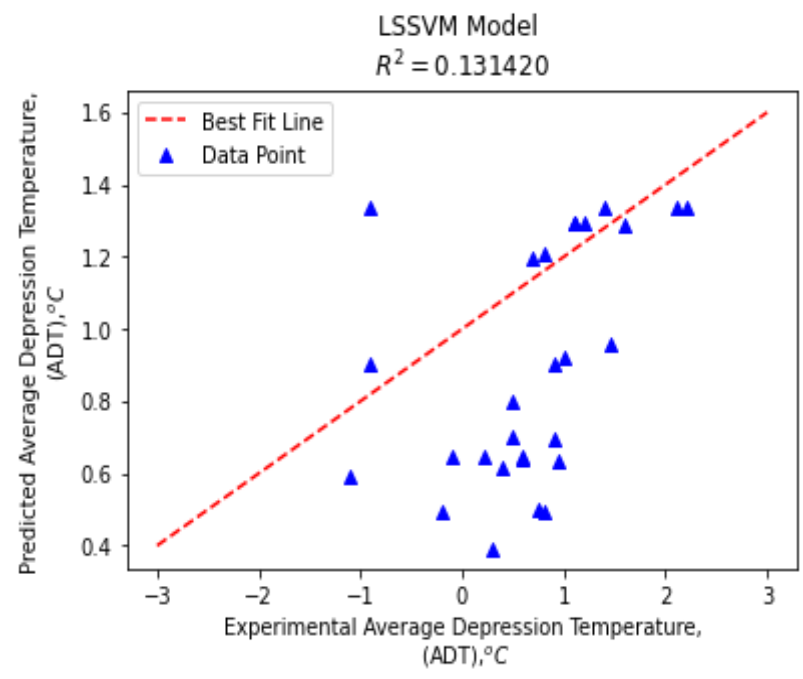

(b)

Fig. 5. Performance of LSSVM model for (a) training and (b) testing sets

From Table 1, it can be justified that GPR model gives highest $\mathrm{R}^{2}$ value of $97.25 \%$ and $96.71 \%$ respectively for training and testing set in comparison to other two models. This shows that GPR model gives higher accuracy in predicting the ADT value depending to the concentration of the inhibitor and the pressure. Mean Square Error (MSE) shows the percentage error of predicted value with the experimental value whereby lower the MSE value, the closer is predicted value to the experimental value. MSE value of GPR for training and testing set gives lowest value of 0.019 and 0.023 respectively compared to ANN and LSSVM model. This signifies that the results of the predicted value of ADT and experimental value of ADT gives low variance with regards to the other ML models leading to lowest prediction error.

Table 1

Error evaluation outcomes for GPR, ANN and LSSVM models

\begin{tabular}{lllllll}
\hline Parameter/Model & GPR & \multicolumn{3}{c}{ ANN } & LSSVM & \\
\hline & Train & Test & Train & Test & Train & Test \\
R $^{2}$ & 97.25 & 96.71 & 96.65 & 95.80 & -0.06 & 0.131 \\
MSE\% & 0.019 & 0.023 & 0.03 & 0.03 & 0.733 & 0.524 \\
\hline
\end{tabular}

\section{Conclusion}

In this research, the advanced risk management technique in preventing the growth of clathrate hydrate in oil and gas pipelines, known as Machine Learning (ML) models that act as predictive analysis, has been extensively applied. In resolving the growth of gas hydrates, there are several ML models, but Gaussian Process Regression (GPR) is the newest method used in minimizing the growth of hydrates in the natural gas industry. The predictive analysis using GPR will be carried out by 
applying the algorithm from the data obtained in terms of pressure, and concentration as the input, while average depression temperature will be the output. To summarize, the ML model that gave the best results in terms of coefficient of determination, $\mathrm{R}^{2}$ and mean squared error, MSE is Gaussian Procession Regression model in comparison to Artificial Neural Network and Least Square Support Vector Machine models. GPR model gave the best results for test and training data in terms of $\mathrm{R}^{2}$ which are $97.25 \%$ and $96.71 \%$ respectively compared to ANN and LSSVM models. MSE value of 0.019 for training set and 0.023 for the testing set obtained is the lowest for GPR model indicating this model gives lowest prediction error of ADT in contrast to other two ML models.

This study is a good example for researchers who want to further understand the correlation between the output which is the ADT and the inputs which are the Pressure and Concentration of the THIs. The researchers will be further understanding the significance of utilizing the ML models to grasp the knowledge of predictive analysis. Nonetheless, it is recommended to obtain more data points before implementing it in this $\mathrm{ML}$ model. This is because ML model are data driven analysis where more experimental work needs to be executed to obtain results that will improve and give more accurate predictive analysis for this ML model especially GPR model that needs large data points. Furthermore, to obtain optimized hyperparameter in the GPR model is another recommendation that needs to be addressed. Further study needs to be developed in understanding the hyperparameter where it is configuration that is external to the model and whose value cannot be estimated from data. This hyperparameters presents mostly in all ML models, especially GPR models hyperparameters such as RBF kernel length-scale value needs to be studied and tuned that will lead to obtaining optimized value of the hyperparameter consequently obtaining precise prediction result of the output.

\section{References}

[1] Bomba, John, Doreen Chin, Ashutosh Kak, and Weihong Meng. "Flow assurance engineering in deepwater offshorepast, present, and future." In Offshore Technology Conference. Offshore Technology Conference, 2018. https://doi.org/10.4043/28704-MS

[2] Khan, Muhammad Saad, Bhajan Lal, Khalik Mohamad Sabil, and Iqbal Ahmed. "Desalination of seawater through gas hydrate process: an overview." Journal of Advanced Research in Fluid Mechanics and Thermal Sciences 55, no. 1 (2019): 65-73.

[3] Qasim, Ali, Jeremy Heurtas, Muhammad Saad Khan, Bhajan Lal, Azmi Mohammad Shariff, Pierre Cezac, Khor Siak Foo, and Jega Divan Sundramoorthy. "Thermodynamic Modeling Of Electrolytic Solutions of Ionic Liquids for Gas Hydrates Inhibition Applications." Journal of Advanced Research in Fluid Mechanics and Thermal Sciences 81, no. 2 (2021): 110-123. https://doi.org/10.37934/arfmts.81.2.110123

[4] Sundramoorthy, Jega Divan, Paul Hammonds, Bhajan Lal, and Greg Phillips. "Gas hydrate gas hydrate equilibrium measurement and observation of gas hydrate dissociation with/without a KHI." Procedia engineering 148 (2016): 870-877. https://doi.org/10.1016/i.proeng.2016.06.476

[5] Kassim, Zamzila, Muhammad Saad Khan, and Bhajan Lal. "Thermodynamic modelling on methane hydrate equilibrium condition in the presence of electrolyte inhibitor." Materials Today: Proceedings 19 (2019): 1395-1402. https://doi.org/10.1016/j.matpr.2019.11.158

[6] Sundramoorthy, Jega Divan, Paul Hammonds, Khalik M. Sabil, Khor Siak Foo, and Bhajan Lal. "Macroscopic observations of catastrophic gas hydrate growth during pipeline operating conditions with or without a kinetic hydrate inhibitor." Crystal Growth \& Design 15, no. $12 \quad$ (2015): 5919-5929. https://doi.org/10.1021/acs.cgd.5b01376

[7] Sahith, Sayani Jai Krishna, Srinivasa Rao Pedapati, and Bhajan Lal. "Investigation on gas hydrates formation and dissociation in multiphase gas dominant transmission pipelines." Applied Sciences 10, no. 15 (2020): 5052. https://doi.org/10.3390/app10155052

[8] Rebai, Noura, Ahmed Hadjadj, Abdelbaki Benmounah, Abdallah S. Berrouk, and Salim M. Boualleg. "Prediction of natural gas hydrates formation using a combination of thermodynamic and neural network modeling." Journal of Petroleum Science and Engineering 182 (2019): 106270. https://doi.org/10.1016/i.petrol.2019.106270 
[9] Yarveicy, Hamidreza, and Mohammad M. Ghiasi. "Modeling of gas hydrate phase equilibria: Extremely randomized trees and LSSVM approaches." Journal of Molecular Liquids 243 (2017): $533-541$. https://doi.org/10.1016/j.molliq.2017.08.053

[10] Qasim, Ali, Muhammad Saad Khan, Bhajan Lal, Mohd Zamri Abdullah, and Abdulhalim Shah Maulud. "Simulation of Hydrate Phase Boundary for Natural Gas Mixture with High $\mathrm{CO}_{2}$ Content through Simulation." International Journal of Engineering and Advanced Technology (IJEAT) 9, no. 2 (2019): 4030-4034. https://doi.org/10.35940/ijeat.B4940.129219

[11] Qasim Ali, Bhajan Lal, Azmi Mohammad Shariff, and Mokhtar Che Ismail. "Role of lonic Liquid-Based Multipurpose Gas Hydrate and Corrosion Inhibitors in Gas Transmission Pipeline." In Nanotechnology-Based Industrial Applications of Ionic Liquids, pp. 221-244. Springer, Cham, 2020. https://doi.org/10.1007/978-3-030-44995-7 11

[12] Khan, Muhammad Saad, Sana Yaqub, Naathiya Manner, Nur Ani Karthwathi, Ali Qasim, Nurhayati Binti Mellon, and Bhajan Lal. "Experimental equipment validation for methane $(\mathrm{CH} 4)$ and carbon dioxide $\left(\mathrm{CO}_{2}\right)$ hydrates." In IOP Conference Series: Materials Science and Engineering, vol. 344, no. 1, p. 012025. IOP Publishing, 2018. https://doi.org/10.1088/1757-899X/344/1/012025

[13] Khan, Muhammad Saad, Cornelius B. Bavoh, Behzad Partoon, Omar Nashed, Bhajan Lal, and Nurhayati Bt Mellon. "Impacts of ammonium based ionic liquids alkyl chain on thermodynamic hydrate inhibition for carbon dioxide rich binary gas." Journal of Molecular Liquids 261 (2018): 283-290. https://doi.org/10.1016/i.molliq.2018.04.015

[14] Rafique, Danish, and Luis Velasco. "Machine learning for network automation: overview, architecture, and applications [Invited Tutorial]." Journal of Optical Communications and Networking 10, no. 10 (2018): D126-D143. https://doi.org/10.1364/JOCN.10.00D126

[15] Jamaludin, Rosmahaida, Nurul Azmira Ibrahim, and Hasmerya Maarof. "Development of structure-activity modelling of carboxamides compounds for Aedes aegypti repellents." Journal of Advanced Research Design 35, no. 1 (2017): 26-32.

[16] Foroozesh, Jalal. "Application of Artificial Intelligence (AI) modeling in kinetics of methane hydrate growth." American Journal of Analytical Chemistry 4, no. 11 (2013): 616-622. https://doi.org/10.4236/ajac.2013.411073

[17] Qin, Hao, Vishal Srivastava, Hua Wang, Luis E. Zerpa, and Carolyn A. Koh. "Machine Learning Models to Predict Gas Hydrate Plugging Risks Using Flowloop and Field Data." In Offshore Technology Conference. OnePetro, 2019. https://doi.org/10.4043/29411-MS

[18] Olajire, Abass A. "Flow assurance issues in deep-water gas well testing and mitigation strategies with respect to gas hydrates deposition in flowlines-A review." Journal of Molecular Liquids (2020): 114203. https://doi.org/10.1016/j.molliq.2020.114203

[19] Bavoh, Cornelius B., Bhajan Lal, Lau Kok Keong, Mazuin binti Jasamai, and Mazlin binti Idress. "Synergic kinetic inhibition effect of EMIM-Cl+ PVP on $\mathrm{CO}_{2}$ hydrate formation." Procedia Engineering 148 (2016): 1232-1238. https://doi.org/10.1016/i.proeng.2016.06.474

[20] Ghiasi, Mohammad M., Hamidreza Yarveicy, Milad Arabloo, Amir H. Mohammadi, and Reza M. Behbahani. "Modeling of stability conditions of natural gas clathrate hydrates using least squares support vector machine approach." Journal of Molecular Liquids 223 (2016): 1081-1092. https://doi.org/10.1016/i.molliq.2016.09.009

[21] Kazemeini, Mohammad, Farideh Freidoonian, and Moslem Fattahi. "Developing a Mathematical Model for Hydrate Formation in a Spray Batch Reactor." Advances in Materials Physics and Chemistry 2, no. 4 (2012): $244-247$. https://doi.org/10.4236/ampc.2012.24B062

[22] Srivastava, Vishal. Quantitative risk modeling of gas hydrate bedding using mechanistic, statistical, and artificial neural network frameworks. Colorado School of Mines, 2018.

[23] Partoon, Behzad, S. Jai Krishna Sahith, Bhajan Lal, and Abdulhalim Shah Bin Maulud. "Gas hydrate models." In Chemical Additives for Gas Hydrates, pp. 67-85. Springer, Cham, 2020. https://doi.org/10.1007/978-3-030-30750$\underline{94}$

[24] Sahith, S. J. Ai Krishna, Srinivasa Rao Pedapati, and Bhajan Lal. "Application of artificial neural networks on measurement of gas hydrates in pipelines." Test Engineering \& Management 81 (2019): 5769-5774.

[25] Song, Yongchen, Hang Zhou, Pengfei Wang, and Mingjun Yang. "Prediction of clathrate hydrate phase equilibria using gradient boosted regression trees and deep neural networks." The Journal of Chemical Thermodynamics 135 (2019): 86-96. https://doi.org/10.1016/i.jct.2019.03.030

[26] Khamehchi, Ehsan, Ebrahim Shamohammadi, and Seyed Hamidreza Yousefi. "Predicting the hydrate formation temperature by a new correlation and neural network." Gas Processing Journal 1, no. 1 (2013): 41-50.

[27] Landgrebe, Michael KB, and Diakanua Nkazi. "Toward a Robust, Universal Predictor of Gas Hydrate Equilibria by Means of a Deep Learning Regression." ACS Omega 4, no. 27 (2019): 22399-22417. https://doi.org/10.1021/acsomega.9b02961

[28] Nasir, Qazi, K. K. Lau, Bhajan Lal, and Khalik M. Sabil. "Hydrate dissociation condition measurement of $\mathrm{CO}^{\mathrm{CO}}{ }_{2}-\mathrm{rich}$ mixed gas in the presence of methanol/ethylene glycol and mixed methanol/ethylene glycol+ electrolyte aqueous 
solution." Journal of Chemical \& Engineering Data 59, no. $11 \quad$ (2014): 3920-3926. https://doi.org/10.1021/je5008313

[29] Soroush, Ebrahim, Mohammad Mesbah, Amin Shokrollahi, Jake Rozyn, Moonyong Lee, Tomoaki Kashiwao, and Alireza Bahadori. "Evolving a robust modeling tool for prediction of natural gas hydrate formation conditions." Journal of Unconventional Oil and Gas Resources 12 (2015): 45-55. https://doi.org/10.1016/j.juogr.2015.09.002

[30] Samsudin, R., P. Saad, and A. Shabri. "River flow time series using least squares support vector machines." Hydrology and Earth System Sciences 15, no. 6 (2011): 1835-1852. https://doi.org/10.5194/hess-15-1835-2011

[31] Çaydaş, Ulaş, and Ahmet Hascalık. "A study on surface roughness in abrasive waterjet machining process using artificial neural networks and regression analysis method." Journal of Materials Processing Technology 202, no. 13 (2008): 574-582. https://doi.org/10.1016/i.jmatprotec.2007.10.024

[32] Sayani, Jai Krishna Sahith, Srinivasa Rao Pedapati, and Bhajan Lal. "Phase behavior study on gas hydrates formation in gas dominant multiphase pipelines with crude oil and high $\mathrm{CO}_{2}$ mixed gas." Scientific Reports 10, no. 1 (2020): 112. https://doi.org/10.1038/s41598-020-71509-6

[33] Saghafi, Hamidreza, and Milad Arabloo. "Modeling of $\mathrm{CO}_{2}$ solubility in MEA, DEA, TEA, and MDEA aqueous solutions using AdaBoost-Decision Tree and Artificial Neural Network." International Journal of Greenhouse Gas Control 58 (2017): 256-265. https://doi.org/10.1016/i.ijggc.2016.12.014

[34] Yazici, Berna, Memmedaga Memmedli, Atilla Aslanargun, and Senay Asma. "Analysis of international debt problem using artificial neural networks and statistical methods." Neural Computing and Applications 19, no. 8 (2010): 12071216. https://doi.org/10.1007/s00521-010-0422-4

[35] Wang, Hu, Enying Li, and Guang Yao Li. "The least square support vector regression coupled with parallel sampling scheme metamodeling technique and application in sheet forming optimization." Materials \& Design 30, no. 5 (2009): 1468-1479. https://doi.org/10.1016/i.matdes.2008.08.014

[36] Qasim, Ali, Muhammad Saad Khan, Bhajan Lal, and Azmi Mohammad Shariff. "A perspective on dual purpose gas hydrate and corrosion inhibitors for flow assurance." Journal of Petroleum Science and Engineering 183 (2019): 106418. https://doi.org/10.1016/i.petrol.2019.106418

[37] Nguyen-Tuong, Duy, Matthias Seeger, and Jan Peters. "Model learning with local gaussian process regression." Advanced Robotics 23, no. 15 (2009): 2015-2034. https://doi.org/10.1163/016918609X12529286896877

[38] Schulz, Eric, Maarten Speekenbrink, and Andreas Krause. "A tutorial on Gaussian process regression: Modelling, exploring, and exploiting functions." Journal of Mathematical Psychology 85 (2018): 1-16. https://doi.org/10.1016/i.jmp.2018.03.001

[39] Bachoc, François, Fabrice Gamboa, Jean-Michel Loubes, and Nil Venet. "A Gaussian process regression model for distribution inputs." IEEE Transactions on Information Theory 64, no. 10 (2017): 6620-6637. https://doi.org/10.1109/TIT.2017.2762322

[40] Kim, Kihwan, Dongryeol Lee, and Irfan Essa. "Gaussian process regression flow for analysis of motion trajectories." In 2011 International Conference on Computer Vision, pp. 1164-1171. IEEE, 2011.

[41] Momeni, Ehsan, Mohammad Bagher Dowlatshahi, Fereydoon Omidinasab, Harnedi Maizir, and Danial Jahed Armaghani. "Gaussian process regression technique to estimate the pile bearing capacity." Arabian Journal for Science and Engineering 45, no. 10 (2020): 8255-8267. https://doi.org/10.1007/s13369-020-04683-4

[42] Kersting, Kristian, Christian Plagemann, Patrick Pfaff, and Wolfram Burgard. "Most likely heteroscedastic Gaussian process regression." In Proceedings of the 24th International Conference on Machine Learning, pp. 393-400. 2007. https://doi.org/10.1145/1273496.1273546

[43] Chen, Tao, Julian Morris, and Elaine Martin. "Gaussian process regression for multivariate spectroscopic calibration." Chemometrics and Intelligent Laboratory Systems 87, no. 1 (2007): 59-71. https://doi.org/10.1016/i.chemolab.2006.09.004

[44] Wang, Bo, and Tao Chen. "Gaussian process regression with multiple response variables." Chemometrics and Intelligent Laboratory Systems 142 (2015): 159-165. https://doi.org/10.1016/i.chemolab.2015.01.016

[45] Hewing, Lukas, Juraj Kabzan, and Melanie N. Zeilinger. "Cautious model predictive control using gaussian process regression." IEEE Transactions on Control Systems Technology 28, no. 6 (2019): $2736-2743$. https://doi.org/10.1109/TCST.2019.2949757

[46] Wilson, Andrew Gordon, David A. Knowles, and Zoubin Ghahramani. "Gaussian process regression networks." In Proceedings of the 29th International Coference on International Conference on Machine Learning, pp. 1139-1146. 2012.

[47] Nashed, Omar, Didar Dadebayev, Muhammad Saad Khan, Cornelius B. Bavoh, Bhajan Lal, and Azmi Mohd Shariff. "Experimental and modelling studies on thermodynamic methane hydrate inhibition in the presence of ionic liquids." Journal of Molecular Liquids 249 (2018): 886-891. https://doi.org/10.1016/i.molliq.2017.11.115 
[48] Khan, Muhammad Saad, Bhajan Lal, Azmi Mohd Shariff, and Hilmi Mukhtar. "Ammonium hydroxide ILs as dualfunctional gas hydrate inhibitors for binary mixed gas (carbon dioxide and methane) hydrates." Journal of Molecular Liquids 274 (2019): 33-44. https://doi.org/10.1016/j.molliq.2018.10.076 